\title{
AEG-1 promotes mesenchymal transition through the activation of Rho GTPases in human glioblastoma cells
}

\author{
SANG-YOON PARK ${ }^{1}$, MINJI CHOI $^{1}$, DAIN PARK $^{2}$, MINHO JEONG $^{3}$, KWANG SEOK AHN $^{1}$, \\ JUNHEE LEE ${ }^{1,2}$, PAUL B. FISHER ${ }^{4}$, MIYONG YUN ${ }^{1,2}$ and SEOK-GEUN LEE ${ }^{1-3}$ \\ ${ }^{1}$ Department of Science in Korean Medicine, College of Korean Medicine, Kyung Hee University; \\ ${ }^{2}$ Korean Medicine Clinical Trial Center, Kyung Hee University Korean Medicine Hospital; \\ ${ }^{3}$ Department of Cancer Preventive Material Development, Graduate School, Kyung Hee University, Seoul 02447, \\ Republic of Korea; ${ }^{4}$ Department of Human and Molecular Genetics, VCU Institute of Molecular Medicine, \\ VCU Massey Cancer Center, Virginia Commonwealth University School of Medicine, Richmond, VA 23298, USA
}

Received April 15, 2016; Accepted May 31, 2016

DOI: $10.3892 /$ or.2016.5106

\begin{abstract}
Despite growing evidence indicating that astrocyte elevated gene-1 (AEG-1) plays pivotal roles in tumor progression in various types of human cancers including brain tumors; to date, its role in the regulation of mesenchymal transition is not clear in glioblastoma. In the present study, we investigated the contribution of AEG-1 to stress fiber formation and then the acquisition of mesenchymal characteristics of glioblastoma cells. Gain- and loss-of-function studies in normal immortalized primary human fetal astrocytes (IM-PHFAs) and glioblastoma cells revealed that overexpression of AEG-1 increased expression of mesenchymal markers including $\mathrm{N}$-cadherin and two mesenchymal transition-inducing transcription factors ZEB1 and Slug but decreased epithelial markers E-cadherin and ZO-1. In addition, knockdown of AEG-1 suppressed invasive ability and migration of glioblastoma cells. Overexpression of AEG-1 also induced stress fiber formation and activated the Rho GTPase signaling pathways in glioblastoma cells. Consistently, treatment with an RhoA inhibitor decreased AEG-1-mediated stress fiber formation in glioblastoma cells. Collectively, our findings suggest that AEG-1 promotes mesenchymal transition in glioblastoma through the regulation of the Rho signaling pathway, resulting in tumor invasion, a primary characteristic of malignant brain tumors.
\end{abstract}

Correspondence to: Professor Seok-Geun Lee or Professor Miyong Yun, Department of Science in Korean Medicine, College of Korean Medicine, Kyung Hee University, 26 Kyungheedae-ro, Dongdaemun-gu, Seoul 02447, Republic of Korea

E-mail: seokgeun@khu.ac.kr

E-mail: myyun91@gmail.com

Key words: AEG-1, mesenchymal transition, invasion, glioblastoma, Rho GTPases

\section{Introduction}

Glioblastoma is the most common malignant tumor in the brain and central nervous system (CNS) with only a 5\% five-year survival rate, and glioblastoma accounts for the majority of gliomas in the USA (1). In Korea, glioblastoma is the most common neuroepithelial tumor accounting for $15.1 \%$ of all primary brain and CNS tumors and $34.4 \%$ of all gliomas (2). Glioblastoma is one of the most difficult cancers to treat, because of the highly invasive nature and the recurrence at new sites of migration (3). In light of the overall poor prognosis of glioblastoma, understanding glioblastoma invasion is crucial for the development of efficient glioblastoma treatment.

Epithelial-mesenchymal transition (EMT) is a necessary event in the invasion and metastasis of various types of human cancers including glioblastoma. EMT is a drastic alteration of epithelial cells involving a shedding of their characteristic morphology and gene expression patterns and the assumption of mesenchymal characteristics including motility which occurs during embryogenesis, wound healing, and cancer progression (4-7). Many cellular changes such as loss of cell junctions and polarity, and reorganization of the cytoskeleton are associated with EMT, resulting in a cellular transformation in terms of morphology, invasiveness and motility (6).

Rho GTPases belong to the Ras superfamily. RhoA, Rac1 and $\mathrm{Cdc} 42$ are critical effectors of actin cytoskeleton reorganization, cellular contraction and migration during the EMT, although the precise molecular mechanisms remain to be elucidated $(8,9)$. According to previous studies, RhoA, Rac1 and $\mathrm{Cdc} 42$ are highly expressed or activated in glioblastoma and promote the invasive behavior of glioma cells (10-16). A most common characteristic of glioblastoma is the overexpression of receptors such as MET and/or EGFR that activate the downstream signaling pathways, PI3K, MAPK and the Rho family of GTPases (17-19). Activated signaling pathways including Rho-GTPases facilitate cancer cell migration and invasion thus inducing metastasis $(20,21)$.

Although astrocyte elevated gene-1 (AEG-1) was first discovered as an HIV-induced gene in primary human fetal astrocytes, studies in the past decade have indicated that 
AEG-1 plays crucial roles in tumor progression such as transformation, survival, invasion, metastasis, angiogenesis and drug resistance in various types of human cancers (22-28). Furthermore, recent reports have revealed that AEG-1 participates in the regulation of the EMT process under diverse cellular conditions (29-35). However, its role in the regulation of EMT in glioblastoma has not been uncovered yet. In the present study, we identified AEG-1 as a mesenchymal transition inducer to mediate invasion and migration of human glioblastoma cells through increasing actin stress fiber formation by activating Rho GTPase signals.

\section{Materials and methods}

Cell cultures. Human glioma cell lines U87MG and U373MG (Korean Cell Line Bank (KCLB), Seoul, Korea), and an immortalized primary human fetal astrocyte cell line IM-PHFA were previously described (36,37). The Lenti-X 293T cell line for lentivirus packaging was purchased from Clontech (Mountain View, CA, USA). All cells were cultured in Dulbecco's modified Eagle's medium (DMEM) supplemented with 10\% heat-inactivated fetal bovine serum (FBS) and $100 \mathrm{U} / \mathrm{ml}$ of antibiotic-antimycotic (both from Lonza, Walkersville, MD, USA) at $37^{\circ} \mathrm{C}$ in a humidified incubator with $5 \% \mathrm{CO}_{2}$, and the viability of the cultured cells was monitored by LUNA-FL Automated Cell Counter (Logos Biosystems, Gyeonggi-do, Korea).

Reagents. 4',6-Diamidino-2-phenylindole (DAPI) and rhodamine-phalloidin were purchased from Invitrogen Life Technologies (Carlsbad, CA, USA). G418 and puromycin were purchased from Biomax (Seoul, Korea). Polybrene was purchased from Santa Cruz Biotechnology (Santa Cruz, CA, USA). Y-27632 was purchased from Sigma-Aldrich. The antibodies used were purchased as following: TCF8/ZEB1, Slug, E-cadherin, N-cadherin, ZO-1, RhoA, Cdc42, Rac1/2/3, phospho-Rac1/Cdc42, and active Rho detection kit from Cell Signaling Technology (Danvers, MA, USA), and $\beta$-actin from Sigma-Aldrich (St. Louis, MO, USA). Horseradish peroxidase-conjugated anti-mouse $\mathrm{IgG}$ and anti-rabbit IgG were purchased from Jackson ImmunoResearch Laboratories, Inc. (West Grove, PA, USA).

Lentivirus production and infection. Lentiviral vectors expressing AEG-1 shRNA were purchased from Sigma-Aldrich (SHCLNG-NM_178812). Control viral vector pLKO.1 GFP shRNA plasmid (Addgene; plasmid \#30323) was a gift from Dr David Sabatini (Massachusetts Institute of Technology, Cambridge, MA, USA), and the envelope vector pMD2.G (plasmid \#12259) and packaging plasmid psPAX2 (plasmid \#12260; both from Addgene) were kindly provided by Dr Didier Trono (Ecole Polytechnique Federale de Lausanne, Lausanne, Switzerland). The lentiviral vector expressing AEG-1 was purchased from OriGene (RC207238L1). Each lentivirus expressing AEG-1 (lenti-AEG-1), AEG-1 shRNA (lenti-AEG-1sh) or GFP shRNA (lenti-GFPsh) was produced by co-transfection with each expression plasmid, pMD2.G and psPAX2 into Lenti-X 293T cells. Transfections were carried out using Lipofector-2000 (AptaBio, Gyeonggi-do, Korea) according to the manufacturer's instructions. Media were harvested at 48, 72 and $96 \mathrm{~h}$ post-transfection and the media were centrifuged at $1,500 \mathrm{rpm}$ for $5 \mathrm{~min}$ and filtered using a $0.45 \mu \mathrm{m}$ syringe filter for removing inadvertently collected cells. Every infection was carried out in the presence of $8 \mu \mathrm{g} / \mathrm{ml}$ of Polybrene. After transduction, cells were selected with $2 \mu \mathrm{g} / \mathrm{ml}$ puromycin.

Western blot analysis and active Rho pull-down assays. Whole cell lysates were prepared, and western blot analysis was performed as previously described (38). Active Rho detection was performed with an active Rho detection kit (Cell Signaling Technology; \#8820) according to the manufacturer's protocol. Seven hundred micrograms of total proteins were used to pull down active Rho with GST-RBD of Rhotekin. Primary antibodies for TCF8/ZEB1 $(1: 1,000)$, Slug (1:1,000), ZO-1 (1:1,000), N-cadherin (1:1,000), E-cadherin (1:1,000), RhoA (1:1,000), Rac1/2/3 (1:1,000), Cdc42 (1:1,000), phospho-Rac1/Cdc42 (1:1,000), active-Rho $(1: 1,000)$ and $\beta$-actin $(1: 10,000)$ were used for immunoblotting followed by horseradish peroxidase-conjugated anti-mouse IgG or antirabbit IgG $(1: 5,000)$ for $1 \mathrm{~h}$, and visualized using the enhanced chemiluminescence detection system.

Phalloidin staining. Cells were plated in 8-well chamber slides and incubated for $24 \mathrm{~h}$ in complete medium. Then the cells were fixed with $4 \%$ paraformaldehyde in PBS for $30 \mathrm{~min}$ and the slides were incubated with $0.1 \%$ Triton $\mathrm{X}-100$ in PBS for $5 \mathrm{~min}$ for permeabilization and blocked with $1 \%$ BSA in PBS for $20 \mathrm{~min}$. Then the cells were incubated with rhodamine-phalloidin for $20 \mathrm{~min}$ and DAPI for $1 \mathrm{~min}$ at room temperature. Images were taken with a FluoView FV1000 confocal microscope (Olympus) and $\mathrm{iRiS}^{\mathrm{TM}}$ Digital Cell Imaging System (Logos Biosystems).

Invasion assays. In vitro invasion assays were performed using 48-well Boyden chambers (Nuero Probe, Inc., Gaithersburg, MD, USA). A polycarbonate membrane with $8-\mu \mathrm{m}$ pore size (Nuero Probe) was coated with Matrigel and dried overnight. The Boyden chambers were filled with medium containing $10 \%$ FBS in the lower compartment, and the coated membrane was mounted in the chamber. Fifty microliters of U87MG cells in serum-free media were placed in the upper chamber of the apparatus and allowed to settle onto the Matrigel-coated membrane. Boyden chambers were incubated at $37^{\circ} \mathrm{C}$ in a $\mathrm{CO}_{2}$ incubator for $16 \mathrm{~h}$. After incubation, the membranes were removed, fixed, and stained with Diff-Quick staining kit. Non-motile cells were removed with cotton swabs from the upper surface of the membrane. Motile cells on the bottom face of the membrane were photographed and the stained cells were counted in three randomly chosen fields using a microscope.

Wound healing assays. U373MG cells were seeded into 6-well plates and incubated for $24 \mathrm{~h}$ until reaching $80-90 \%$ confluency. A $200-\mu 1$ pipette tip was used to make scratches in each well. Several regions were marked and photographed at 0 and $24 \mathrm{~h}$ after the scratches were made. Phase-contrast microscopy images were taken using Zeiss Axio. Cell motility was calculated as the area covered by the cells between the edges at the time of measurement. Migration rate $=$ (mean area occupied by cells/mean original area) x 100. Each test group was assayed in triplicate at least. 


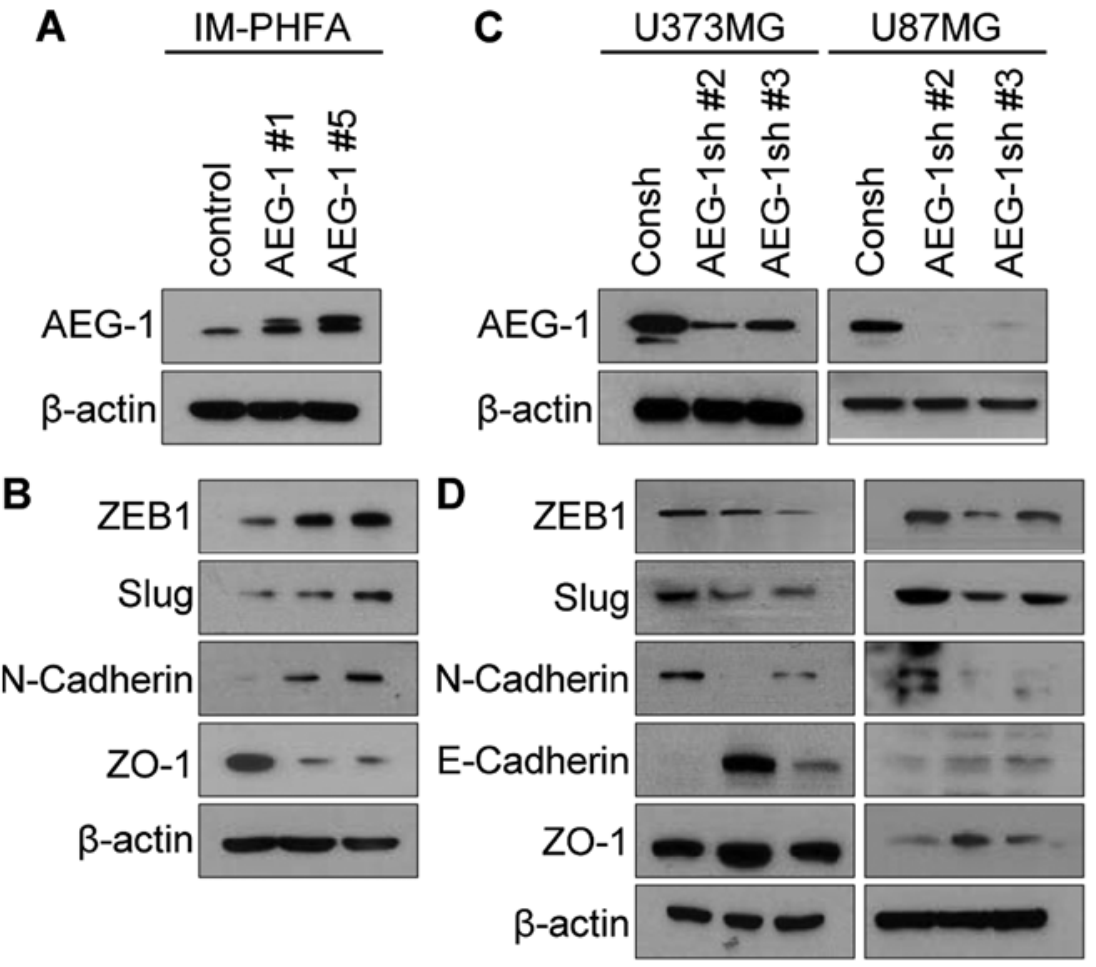

Figure 1. AEG-1 induces mesenchymal transition of glioblastoma cells. (A and B) IM-PHFA cells were stably transfected with lenti-control or lenti-AEG-1. Cell lysates were prepared, and then subjected to western blot analysis with the indicated antibodies. (C and D) U373MG and U87MG cells were infected with lenti-GFPsh (Consh) or lenti-AEG-1sh (AEG-1sh). Cell lysates were prepared, and then subjected to western blot analysis with the indicated antibodies. $\beta$-actin was used as an internal control. AEG-1, astrocyte elevated gene-1; IM-PHFA, immortalized primary human fetal astrocyte cell line.

Statistical analysis. Data are presented as the mean \pm standard error of the mean (SEM) and were analyzed for statistical significance using the unpaired Student's t-test. $\mathrm{P}<0.05$ was considered statistically significant.

\section{Results}

AEG-1 induces mesenchymal markers in human glioblastoma cells. To examine whether AEG-1 promotes mesenchymal transition of glioblastoma cells, we modulated AEG-1 expression in normal astrocytes (IM-PHFA), and glioblastoma cells (U373MG and U87MG) (Fig. 1A and C). Overexpression of AEG-1 in IM-PHFA cells intensively induced expression levels of the mesenchymal markers ZEB1, Slug and N-cadherin, but reduced expression of the epithelial marker ZO-1 (Fig. 1B). Consistently knockdown of AEG-1 in human glioblastoma cells decreased expression of mesenchymal markers ZEB1, Slug and N-cadherin, but increased expression of epithelial markers E-cadherin and ZO-1 (Fig. 1D). These results indicate that AEG-1 may promote mesenchymal transition of human glioblastoma cells.

Knockdown of AEG-1 suppresses invasion and migration of human glioblastoma cells. Since mesenchymal transition of glioblastoma is involved in the invasion and migration of cancer cells, we then aimed to ascertain whether AEG-1 affects invasive ability and motility of human glioblastoma cells. As shown in Fig. 2A and B, knockdown of AEG-1 in glioblastoma cells decreased more than $58 \%$ of invaded cells compared with the control. In addition, wound healing assays showed that knockdown of AEG-1 significantly inhibited the migration of glioblastoma cells (Fig. 2C and D). Collectively, these results suggest that AEG-1 may play an important role in the invasion and migration of glioblastoma cells through the induction of mesenchymal transition.

AEG-1 induces formation of stress fibers in human glioblastoma cells. Actin stress fibers that induce cytoskeleton remodeling are associated with cellular contractility providing force for increased cell movement during mesenchymal transition (39). Thus, we examined whether AEG-1 modulates stress fiber formation. Overexpression of AEG-1 in IM-PHFA cells significantly increased actin stress fibers (Fig. 3A). In accordance, knockdown of AEG1 in glioblastoma cells dramatically decreased stress fibers (Fig. 3B). These results indicate that AEG-1 increases actin stress fiber formation to induce changes in actin cytoskeleton structures and the motility of human glioblastoma cells.

AEG-1 activates Rho family GTPases in human glioblastoma cells. During mesenchymal transition, cells require dynamic actin rearrangement and cytoskeleton remodeling via regulation of Rho family GTPases (39). Overexpression of AEG-1 in IM-PHFA cells intensively increased the active form of RhoA (RhoA-GTP) and phosphorylation of Rac1 and $\mathrm{Cdc} 42$, and also increased the protein levels of the Rho family GTPases (Fig. 4A). Knockdown of AEG-1 in glioblastoma cells significantly reduced expression of the Rho family proteins as well as their activation (Fig. 4A). Rho-associated, coiled-coil-containing protein kinase 1 (ROCK1) is a major downstream protein of RhoA, which promotes F-actin stabilization and induction of Rac signaling (39). To confirm whether 


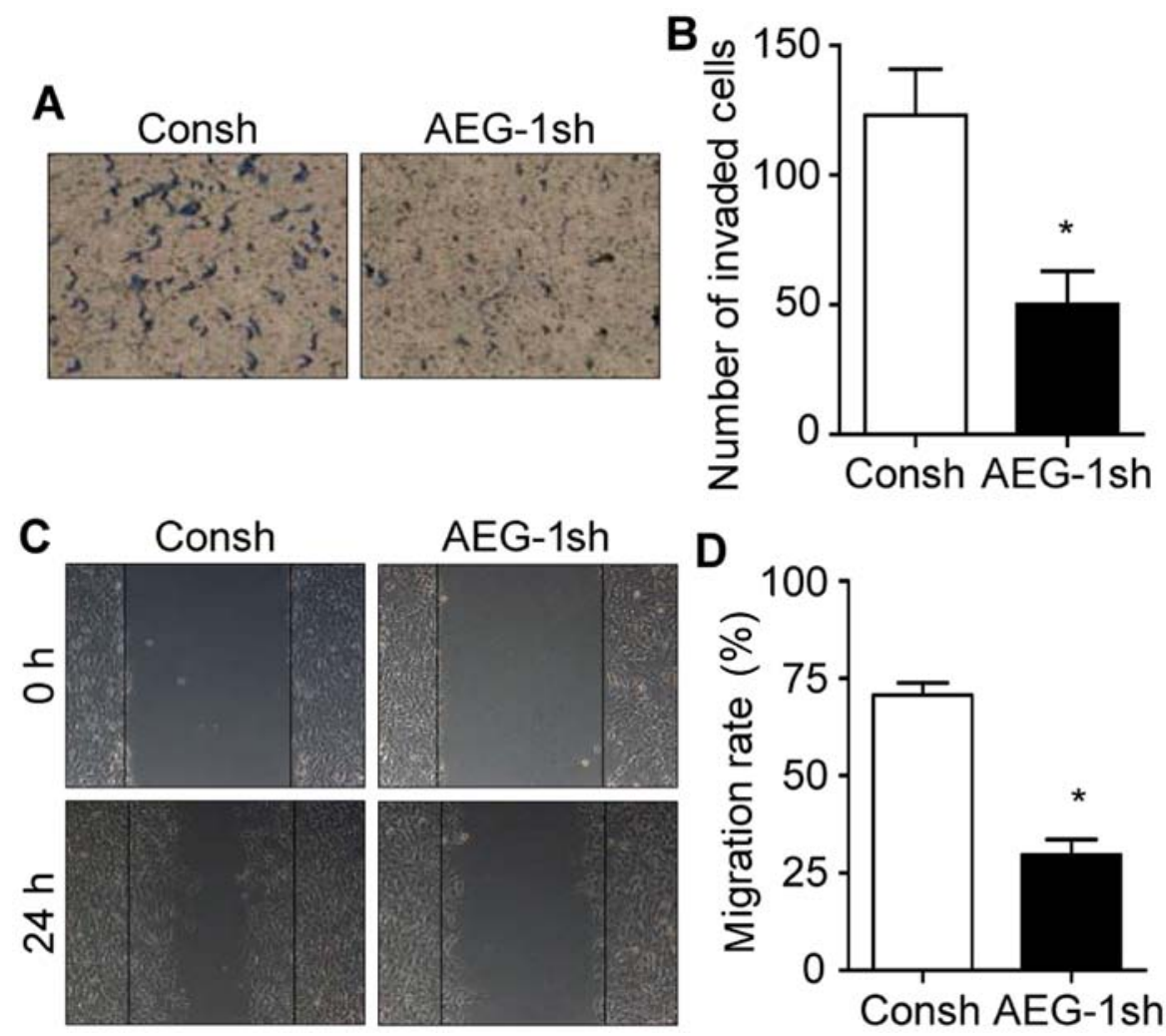

Figure 2. Knockdown of AEG-1 decreases invasion and migration of glioblastoma cells. (A) U87MG-AEG-1sh cells were seeded onto the upper chamber of Boyden chambers in the absence of serum, and $16 \mathrm{~h}$ after seeding the membranes were fixed, stained and photographed. U87MG-AEG-1sh cells showed a significant decrease in invasive ability compared with that of the U87MG-Consh cells. (B) Quantitative analyses of the invaded cells. (C) U373MG-AEG-1sh cells were seeded onto 6-well plates and scratched using a 200- $\mu \mathrm{l}$ pipette tip and photographed at 0 and $24 \mathrm{~h}$. (D) The quantitative graph indicates the wound area covered with migrated cells at $24 \mathrm{~h}$ as a percentage of the area at $0 \mathrm{~h} .{ }^{~} \mathrm{P}<0.05$ compared to the control group. AEG-1, astrocyte elevated gene-1.

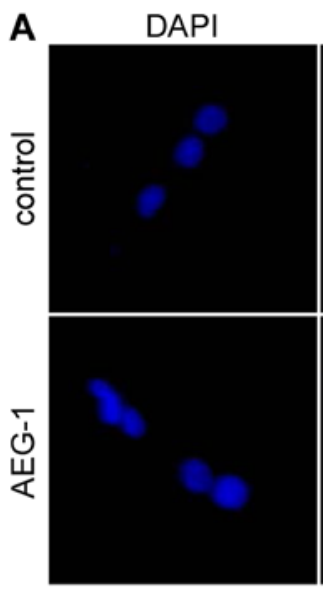

B

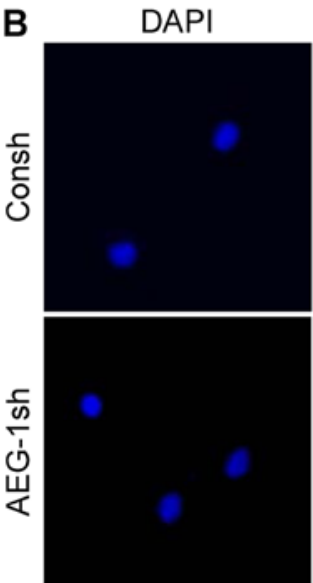

Phalloidin

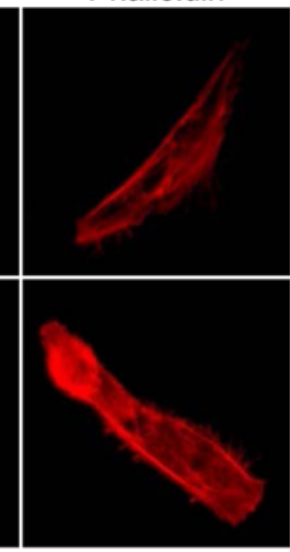

Phalloidin

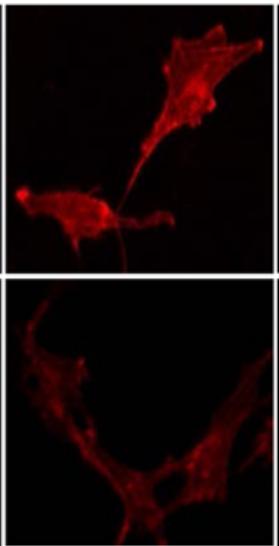

Merge
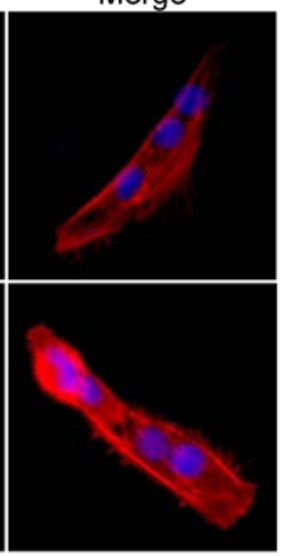

Merge

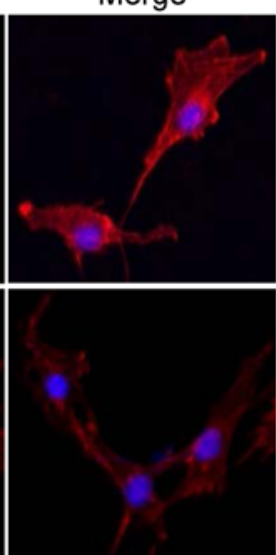

AEG-1-induced RhoA activation is critical for mesenchymal transition, we used Y-27632 a specific inhibitor of ROCK1. As shown in Fig. 4B, treatment with Y-27632 attenuated AEG-1induced stress fibers. Collectively these results revealed that AEG-1 activates Rho signals to induce stress fiber formation and mesenchymal transition.

\section{Discussion}

Glioblastoma is commonly classified as a malignant form of glioma with highly invasive behavior and a poor survival rate and arises from astrocytes. The invasive ability of glioblastoma into the brain parenchyma is the major obstacle to treatment, because tumor cells can evade surgical resection and radiation therapy (3). Although diffuse invasion of malignant glioma is one of its most adverse characteristics and thus has been vigorously investigated, relatively few mechanisms involved in the induction of mesenchymal transition and invasion of glioma cells have been uncovered.

Figure 3. AEG-1 induces the formation of actin stress fibers in glioblastoma cells. (A) IM-PHFA cells were infected with lenti-control or lenti-AEG-1. (B) U373MG cells were infected with lenti-GFPsh (Consh) or lenti-AEG-1sh (AEG-1sh). DAPI and rhodamine-phalloidin were used to stain the nucleus and the actin cytoskeleton, respectively. AEG-1, astrocyte elevated gene-1; IM-PHFA, immortalized primary human fetal astrocyte cell line; DAPI, 4',6-diamidino-2-phenylindole. 
A
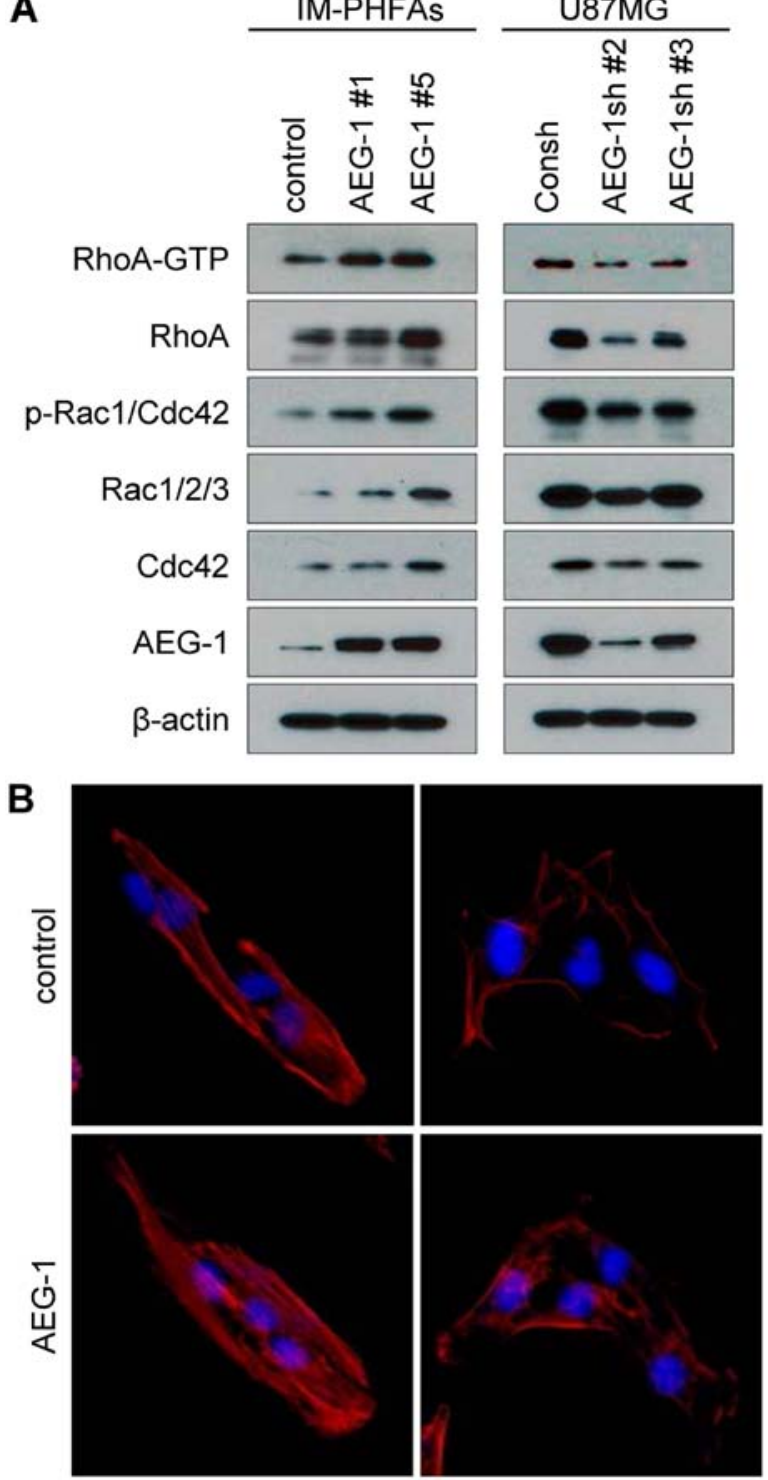

vehicle

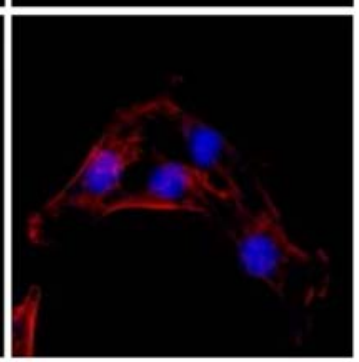

Y-27632

Figure 4. AEG-1 induces Rho signaling pathways in glioblastoma cells. (A) IM-PHFA cells were infected with lenti-control or lenti-AEG-1. U87MG cells were infected with lenti-GFPsh (Consh) or lenti-AEG-1sh. Cell lysates were prepared, and then subjected to western blot analysis with the indicated antibodies. $\beta$-actin was used as an internal control. For active Rho detection, cell lysates were subjected to pull-down assay using GST-RBD and immunoblotting with an active-Rho antibody. (B) IM-PHFA-AEG-1 cells were incubated with Y-27632 $10 \mu \mathrm{M}$ for $30 \mathrm{~min}$. The F-actin of the cells was then stained with rhodamine-phalloidin. AEG-1, astrocyte elevated gene-1; IM-PHFA, immortalized primary human fetal astrocyte cell line.

Since AEG-1 was initially identified as an HIV-1-inducible gene, evidence that AEG-1 is related with cancer progression and thus functions as an oncogene has accumulated (40-42). AEG-1 is elevated in various types of human cancers including carcinomas of the liver, gallbladder, kidney, breast, lung, prostate, ovary, esophagus, stomach and colon, as well as glioma, neuroblastoma, melanoma, and osteosarcoma $(40,43)$. Increased AGE-1 in various types of human cancers participates in diverse signaling pathways such as the PI3K/AKT, $\mathrm{NF}-\kappa \mathrm{B}, \mathrm{Wnt} / \beta$-catenin and MAPK pathways leading to proliferation, survival, drug resistance, EMT, invasion and

metastasis of cancer (44). Particularly, AEG-1 plays crucial roles during EMT in hepatocellular carcinoma, non-small cell lung cancer and cervical cancer by activating diverse signals such as Wnt/ $\beta$-catenin and TGF- $\beta$ (29-35). In the present study we revealed that AEG-1 also participated in the induction of mesenchymal transition and then invasion and motility of glioblastoma.

Dissociation of cell-cell junctions, loss of polarity, reorganization of the cytoskeletal structure, acquisition of a front-rear polarity, and increase in motility are key events during EMT $(45,46)$. Alteration of the cytoskeletal architecture results in stress fiber formation that is one of the common EMT markers (47). Induction of actin dynamics and rearrangement require activation of the Rho family of proteins (48). RhoA, Rac1 and Cdc42 play crucial roles in the formation of actin stress fiber, lamellipodia and filopodia, respectively (49-51). As shown in Figs. 3 and 4, AEG-1 promotes actin stress fiber formation by activating RhoA in glioblastoma cells. In addition, AEG-1 induces activation of RhoA, Rac1 and Cdc42 as well as their expression. These results indicate that AEG-1 regulates the actin cytoskeleton structure by modulating the Rho family of proteins in human glioblastoma cells.

Collectively, these results suggest that AEG-1 promotes mesenchymal transition in human glioblastoma cells through increasing formation of actin stress fibers by activation and also by the induction of the Rho family of proteins. This may be an important mechanism by which AEG-1 induces diffuse invasion of glioblastoma into the brain parenchyma. Therefore, further studies to investigate the role of AEG-1 in the formation of lamellipodia and filopodia in glioblastoma are warranted to reveal the more detailed mechanisms of glioblastoma invasion and motility and then to pave the way for developing novel therapeutic interventions and ameliorating the suffering of glioblastoma patients from this most aggressive and fatal disease.

\section{Acknowledgments}

This study was supported by research grants from the National Research Foundation of Korea (grant nos. 2007-0054931, NRF-2013R1A1A2007263 and NRF-2013R1A2A2A01069099) and from the National R\&D Program for Cancer Control of the Ministry of Health and Welfare, Republic of Korea (grant no. 1320120).

\section{References}

1. Ostrom QT, Gittleman H, Liao P, Rouse C, Chen Y, Dowling J, Wolinsky Y, Kruchko C and Barnholtz-Sloan J: CBTRUS statistical report: Primary brain and central nervous system tumors diagnosed in the United States in 2007-2011. Neuro-oncol 16 (Suppl 4): iv1-iv63, 2014.

2. Jung KW, Ha J, Lee SH, Won YJ and Yoo H: An updated nationwide epidemiology of primary brain tumors in Republic of Korea. Brain Tumor Res Treat 1: 16-23, 2013.

3. Cuddapah VA, Robel S, Watkins S and Sontheimer H: A neurocentric perspective on glioma invasion. Nat Rev Neurosci 15: 455-465, 2014.

4. Chapman HA: Epithelial-mesenchymal interactions in pulmonary fibrosis. Annu Rev Physiol 73: 413-435, 2011.

5. Huang RY, Guilford P and Thiery JP: Early events in cell adhesion and polarity during epithelial-mesenchymal transition. J Cell Sci 125: 4417-4422, 2012.

6. Kalluri R and Weinberg RA: The basics of epithelial-mesenchymal transition. J Clin Invest 119: 1420-1428, 2009. 
7. Thiery JP, Acloque H, Huang RY and Nieto MA: Epithelialmesenchymal transitions in development and disease. Cell 139: 871-890, 2009.

8. Haudenschild DR, D'Lima DD and Lotz MK: Dynamic compression of chondrocytes induces a Rho kinase-dependent reorganization of the actin cytoskeleton. Biorheology 45: 219-228, 2008.

9. Nobes CD and Hall A: Rho, Rac, and Cdc42 GTPases regulate the assembly of multimolecular focal complexes associated with actin stress fibers, lamellipodia, and filopodia. Cell 81: 53-62, 1995

10. Bigarella CL, Borges L, Costa FF and Saad ST: ARHGAP21 modulates FAK activity and impairs glioblastoma cell migration. Biochim Biophys Acta 1793: 806-816, 2009.

11. Fortin SP, Ennis MJ, Schumacher CA, Zylstra-Diegel CR, Williams BO, Ross JT, Winkles JA, Loftus JC, Symons MH and Tran NL: Cdc42 and the guanine nucleotide exchange factors Ect 2 and trio mediate Fn14-induced migration and invasion of glioblastoma cells. Mol Cancer Res 10: 958-968, 2012.

12. Johnston AL, Lun X, Rahn JJ, Liacini A, Wang L, Hamilton MG, Parney IF, Hempstead BL, Robbins SM, Forsyth PA, et al: The p75 neurotrophin receptor is a central regulator of glioma invasion. PLoS Biol 5: e212, 2007.

13. Malchinkhuu E, Sato K, Maehama T, Mogi C, Tomura H, Ishiuchi S, Yoshimoto Y, Kurose H and Okajima F: $\mathrm{S}_{1} \mathrm{P}_{2}$ receptors mediate inhibition of glioma cell migration through Rho signaling pathways independent of PTEN. Biochem Biophys Res Commun 366: 963-968, 2008.

14. Salhia B, Tran NL, Chan A, Wolf A, Nakada M, Rutka F, Ennis M, McDonough WS, Berens ME, Symons M, et al: The guanine nucleotide exchange factors trio, Ect2, and Vav3 mediate the invasive behavior of glioblastoma. Am J Pathol 73: 1828-1838, 2008.

15. Tran NL, McDonough WS, Savitch BA, Fortin SP, Winkles JA, Symons M, Nakada M, Cunliffe HE, Hostetter G, Hoelzinger DB, et al: Increased fibroblast growth factor-inducible 14 expression levels promote glioma cell invasion via Racl and nuclear factor-kappaB and correlate with poor patient outcome. Cancer Res 66: 9535-9542, 2006.

16. Yan B, Chour HH, Peh BK, Lim C and Salto-Tellez M: RhoA protein expression correlates positively with degree of malignancy in astrocytomas. Neurosci Lett 407: 124-126, 2006.

17. Fortin Ensign SP, Mathews IT, Symons MH, Berens ME and Tran NL: Implications of Rho GTPase signaling in glioma cell invasion and tumor progression. Front Oncol 3: 241, 2013.

18. Kwiatkowska A and Symons M: Signaling determinants of glioma cell invasion. Adv Exp Med Biol 986: 121-141, 2013.

19. Nakada M, Nakada S, Demuth T, Tran NL, Hoelzinger DB and Berens ME: Molecular targets of glioma invasion. Cell Mol Life Sci 64: 458-478, 2007

20. Chang L and Goldman RD: Intermediate filaments mediate cytoskeletal crosstalk. Nat Rev Mol Cell Biol 5: 601-613, 2004.

21. Helfand BT, Chang L and Goldman RD: Intermediate filaments are dynamic and motile elements of cellular architecture. J Cell Sci 117: 133-141, 2004.

22. Emdad L, Lee SG, Su ZZ, Jeon HY, Boukerche H, Sarkar D and Fisher PB: Astrocyte elevated gene-1 (AEG-1) functions as an oncogene and regulates angiogenesis. Proc Natl Acad Sci USA 106: 21300-21305, 2009.

23. Gnosa S, Shen YM, Wang CJ, Zhang H, Stratmann J, Arbman G and Sun XF: Expression of AEG-1 mRNA and protein in colorectal cancer patients and colon cancer cell lines. J Trans Med 10: 109, 2012.

24. Gnosa S, Zhang H, Brodin VP, Carstensen J, Adell G and Sun XF: AEG-1 expression is an independent prognostic factor in rectal cancer patients with preoperative radiotherapy: A study in a Swedish clinical trial. Br J Cancer 111: 166-173, 2014.

25. Jiang T, Zhu A, Zhu Y and Piao D: Clinical implications of AEG-1 in liver metastasis of colorectal cancer. Med Oncol 29: 2858-2863, 2012

26. Ke ZF, He S, Li S, Luo D, Feng C and Zhou W: Expression characteristics of astrocyte elevated gene-1 (AEG-1) in tongue carcinoma and its correlation with poor prognosis. Cancer Epidemiol 37: 179-185, 2013

27. Ke ZF, Mao X, Zeng C, He S, Li S and Wang LT: AEG-1 expression characteristics in human non-small cell lung cancer and its relationship with apoptosis. Med Oncol 30: 383, 2013.

28. Li C, Liu J, Lu R, Yu G, Wang X, Zhao Y, Song H, Lin P, Sun X, Yu X, et al: AEG-1 overexpression: A novel indicator for peritoneal dissemination and lymph node metastasis in epithelial ovarian cancers. Int J Gynecol Cancer 21: 602-608, 2011.
29. He W, He S, Wang Z, Shen H, Fang W, Zhang Y, Qian W, Lin M, Yuan J, Wang J, et al: Astrocyte elevated gene-1(AEG-1) induces epithelial-mesenchymal transition in lung cancer through activating Wnt $/ \beta$-catenin signaling. BMC Cancer 15: 107, 2015.

30. Li WN, Wei JL, Wu M, Wu W, Huang Y, Xie MW and Han H: AEG-1 participates in high glucose-induced activation of Rho kinase and epithelial-mesenchymal transition in proximal tubular epithelial cells. Asian Pac J Trop Med 8: 1076-1078, 2015.

31. Liu K, Guo L, Guo Y, Zhou B, Li T, Yang H, Yin R and Xi T: AEG-1 3'-untranslated region functions as a ceRNA in inducing epithelial-mesenchymal transition of human non-small cell lung cancer by regulating miR-30a activity. Eur J Cell Biol 94: 22-31, 2015.

32. Song E, Yu W, Xiong X, Kuang X, Ai Y and Xiong X: Astrocyte elevated gene-1 promotes progression of cervical squamous cell carcinoma by inducing epithelial-mesenchymal transition via Wnt signaling. Int J Gynecol Cancer 25: 345-355, 2015.

33. Wei J, Li Z, Chen W, Ma C, Zhan F, Wu W and Peng Y: AEG-1 participates in TGF-beta1-induced EMT through p38 MAPK activation. Cell Biol Int 37: 1016-1021, 2013.

34. Zhang J, Zhu D, Lv Q, Yi Y, Li F and Zhang W: The key role of astrocyte elevated gene-1 in CCR6-induced EMT in cervical cancer. Tumour Biol 36: 9763-9767, 2015.

35. Zheng J, Li C, Wu X, Yang Y, Hao M, Sheng S, Sun Y, Zhang H, Long J and $\mathrm{Hu} \mathrm{C}$ : Astrocyte elevated gene-1 is a novel biomarker of epithelial-mesenchymal transition and progression of hepatocellular carcinoma in two China regions. Tumour Biol 35: 2265-2269, 2014.

36. Lee SG, Su ZZ, Emdad L, Sarkar D, Franke TF and Fisher PB: Astrocyte elevated gene-1 activates cell survival pathways through PI3K-Akt signaling. Oncogene 27: 1114-1121, 2008.

37. Park D, Ha IJ, Park SY, Choi M, Lim SL, Kim SH, Lee JH, Ahn KS, Yun M and Lee SG: Morusin induces TRAIL sensitization by regulating EGFR and DR5 in human glioblastoma cells. J Nat Prod 79: 317-323, 2016

38. Lee SG, Kim K, Kegelman TP, Dash R, Das SK, Choi JK, Emdad L, Howlett EL, Jeon HY, Su ZZ, et al: Oncogene AEG-1 promotes glioma-induced neurodegeneration by increasing glutamate excitotoxicity. Cancer Res 71: 6514-6523, 2011

39. Lamouille S, Xu J and Derynck R: Molecular mechanisms of epithelial-mesenchymal transition. Nat Rev Mol Cell Biol 15: 178-196, 2014.

40. Lee SG, Kang DC, DeSalle R, Sarkar D and Fisher PB: AEG-1/MTDH/LYRIC, the beginning: Initial cloning, structure, expression profile, and regulation of expression. Adv Cancer Res 120: 1-38, 2013.

41. Sarkar D and Fisher PB: Advances in Cancer Research. AEG-1/MTDH/LYRIC implicated in multiple human cancers. Preface. Adv Cancer Res 120: xi-xiv, 2013.

42. Su ZZ, Kang DC, Chen Y, Pekarskaya O, Chao W, Volsky DJ and Fisher PB: Identification and cloning of human astrocyte genes displaying elevated expression after infection with HIV-1 or exposure to HIV-1 envelope glycoprotein by rapid subtraction hybridization, RaSH. Oncogene 21: 3592-3602, 2002.

43. Huang Y and Li LP: Progress of cancer research on astrocyte elevated gene-1/Metadherin (Review). Oncol Lett 8: 493-501, 2014.

44. Emdad L, Das SK, Dasgupta S, Hu B, Sarkar D and Fisher PB: AEG-1/MTDH/LYRIC: Signaling pathways, downstream genes, interacting proteins, and regulation of tumor angiogenesis. Adv Cancer Res 120: 75-111, 2013.

45. Hay ED: An overview of epithelial-mesenchymal transformation. Acta Anat (Basel) 154: 8-20, 1995.

46. Thiery JP and Sleeman JP: Complex networks orchestrate epithelial-mesenchymal transitions. Nat Rev Mol Cell Biol 7: 131-142, 2006.

47. Haynes J, Srivastava J, Madson N, Wittmann T and Barber DL: Dynamic actin remodeling during epithelial-mesenchymal transition depends on increased moesin expression. Mol Biol Cell 22: 4750-4764, 2011

48. Jaffe AB and Hall A: Rho GTPases: Biochemistry and biology. Annu Rev Cell Dev Biol 21: 247-269, 2005.

49. Etienne-Manneville S and Hall A: Rho GTPases in cell biology. Nature 420: 629-635, 2002.

50. Ridley AJ: Rho GTPases and actin dynamics in membrane protrusions and vesicle trafficking. Trends Cell Biol 16: 522-529, 2006.

51. Valencia A, Chardin P, Wittinghofer A and Sander C: The ras protein family: Evolutionary tree and role of conserved amino acids. Biochemistry 30: 4637-4648, 1991. 